\title{
Survival and Functional Outcome after Treatment for Primary Base of Tongue Cancer: A Comparison of Definitive Chemoradiotherapy versus Surgery Followed by Adjuvant Radiotherapy
}

\author{
Sangjoon Park, MD ${ }^{1}$ \\ Yeona Cho, MD ${ }^{1}$ \\ Jeongshim Lee, MD' \\ Yoon Woo Koh, MD, PhD² \\ Se-Heon Kim, MD, PhD² \\ Eun Chang Choi, MD, PhD² \\ Hye Ryun Kim, MD, PhD ${ }^{3}$ \\ Ki Chang Keum, MD, PhD' \\ Kyung Ran Park, MD, PhD ${ }^{4}$ \\ Chang Geol Lee, MD, $\mathrm{PhD}{ }^{1}$
}

Departments of ${ }^{1}$ Radiation Oncology,

${ }^{2}$ Otorhinolaryngology, and

${ }^{3}$ Medical Oncology, Yonsei University

College of Medicine, Seoul, Korea,

${ }^{4}$ Department of Radiation Oncology,

University of Washington Medical Center,

Seattle, WA, USA

\author{
Correspondence: Chang Geol Lee, MD, PhD \\ Department of Radiation Oncology, \\ Yonsei Cancer Center, Yonsei University \\ College of Medicine, 50 Yonsei-ro, \\ Seodaemun-gu, Seoul 03722, Korea \\ Tel: 82-2-2228-8114 \\ Fax: 82-2-312-9033 \\ E-mail: CGLEE1023@yuhs.ac \\ Received October 18, 2017 \\ Accepted December 22, 2017 \\ Published Online December 26, 2017
}

\begin{abstract}
Purpose
The purpose of this study was to compare the clinical and functional outcomes in patients with primary base of tongue (BOT) cancer who received definitive radiotherapy (RT) or surgery followed by radiotherapy (SRT).
\end{abstract}

\section{Materials and Methods}

Between January 2002 and December 2016, 102 patients with stage I-IVB primary BOT cancer underwent either definitive RT ( $n=46)$ or SRT ( $n=56)$, and treatment outcomes were compared between two groups. The expression of p16 was also analyzed.

\section{Results}

The RT group had more patients with advanced T stage (T3-4) disease (58.7\% vs. 35.7\%, $p=0.021)$ and who received chemotherapy $(91.3 \%$ vs. $37.5 \%, p<0.001)$ than the SRT group. At a median follow up of 36.9 months (range, 3.3 to 181.5 months), the 5 -year overall survival (OS) and disease-free survival (DFS) were $75.5 \%$ and $68.7 \%$, respectively. With respect to treatment group, the 5-year OS and DFS in the RT and SRT groups did not differ significantly (OS, $68.7 \%$ vs. $80.5 \%, p=0.601$; DFS, $63.1 \%$ vs. $73.1 \%, p=0.653$ ). In multivariate analysis, OS differed significantly according to $\mathrm{p} 16$ expression (p16-negative vs. p16positive; hazard ratio [HR], 0.145; 95\% confidence interval [Cl], 0.025 to $0.853 ; p=0.033$ ). Regarding DFS, p16 expression (p16-negative vs. p16-positive; HR, 0.164; 95\% Cl, 0.045 to $0.598 ; p=0.006$ ) showed a significant effect in multivariate analysis. Functional defects (late grade $\geq 3$ dysphagia or voice alteration) were more frequently reported in the SRT than in the RT group $(16.1 \%$ vs. $2.2 \%, p=0.021)$.

\section{Conclusion}

Despite advanced disease, patients in the RT group showed comparable survival outcomes and better functional preservation than those in the SRT group.

\section{Introduction}

Until recently, the treatment of base of tongue (BOT) cancer has been a challenge for head and neck surgeons, radiation oncologists, and medical oncologists. Patients with this cancer are often diagnosed in advance stages because of its ambiguous symptoms [1]. In cases of radical surgery, serious functional impairment occurs in the majority of cases; even after radical resection, the recurrence rate is high [2,3].

\section{Key words}

Base of tongue cancer, Radiotherapy, Surgery,

Treatment outcome, Organ preservation

The current standard for treatment of BOT cancer is either surgery or definitive radiotherapy (RT) with or without concurrent chemotherapy $[4,5]$. However, no published randomized studies have directly compared the efficacy of these treatment options due to the low incidence rate of BOT cancer. Therefore, the treatment method is determined according to the preference of the patient and the physician considering the cancer stage, possibility of complete excision, and functional loss expected after treatment.

There have been considerable advances in RT and surgical 
techniques and both treatments are expected to provide better results $[6,7]$. In this context, research is needed to determine the optimal treatment for primary BOT cancer.

The purpose of this study was to compare the clinical and functional outcomes in patients with BOT cancer who received either definitive RT or surgery followed by postoperative RT (SRT).

\section{Materials and Methods}

\section{Patients}

Data from a total of 139 consecutive patients who received definitive RT or postoperative RT after primary surgery for BOT squamous cell carcinoma between January 2001 and December 2016, were retrospectively reviewed. Patients with double primary cancer $(n=4)$, distant metastasis $(n=1)$, re-irradiation $(n=2)$, incomplete $\mathrm{RT}(\mathrm{n}=5)$, histologic type other than squamous cell carcinoma $(n=22)$, or without image follow-up $(n=3)$ were excluded, leaving 102 patients included in the analyses. Pretreatment evaluation, including a complete history and physical examination, endoscopic examination, computed tomography (CT), and magnetic resonance imaging was performed for every patient. Patients were staged according to the 2010 American Joint Committee on Cancer criteria (7th edition). Patients treated with definitive RT were staged clinically, whereas those with primary surgery were staged both pathologically and clinically.

\section{Treatments}

When selecting the optimal treatment strategy in our institution, clinicians consider the tumor extent and location; pharyngeal function; and patient age, medical co-morbidities, and preferences. As a result, 56 patients received surgery followed by postoperative RT (SRT group), while 43 patients were treated with definitive RT (RT group).

The surgical techniques included local or composite resection either by open approaches or by transoral robotic surgery (TORS) using da Vinci Robot (Intuitive Surgical Inc., Sunnyvale, CA). In those who received surgery, neck dissection was performed on the involved side or both sides of the neck.

In every patient, CT-based RT planning was used and RT was performed using either 3-dimensional conformal RT or intensity-modulated RT (IMRT). The median RT doses were $63 \mathrm{~Gy}$ (range, 52 to $68.4 \mathrm{~Gy}$ ) and $70 \mathrm{~Gy}$ (range, 63 to $76 \mathrm{~Gy}$ ) for the SRT and RT groups, respectively. The RT field was defined as the primary tumor with sufficient margins and both sides of the neck. Gross tumors or involved lymph nodes were treated with 66-70 Gy, postoperative tumors or nodal beds with 60-66 Gy, involved nodal stations with 60-63 Gy, and uninvolved nodal stations with 45-50 Gy.

Concurrent chemotherapy was performed with either cisplatin or cetuximab-based regimens. The induction chemotherapy regimen consisted of the combination of docetaxel, cisplatin, 5-fluorouracil, or titanium silicate-1, and cisplatin.

\section{Outcome assessment}

Patients without follow-up imaging were censored from the analysis. Treatment failures were defined as persistent or recurrent loco-regional disease or distant metastases. The date of tissue confirmation or imaging study showing evidence of failure was considered the date of failure. Acute toxicities were defined as adverse events occurring within 90 days from the start of treatment, while late toxicities were defined as those observed at least 90 days after the start of treatment. The severity of toxicities was scored using Common Terminology Criteria for Adverse Events (CTCAE) ver. 4.0 and toxicities $\geq$ grade 3 were recorded. Among late toxicities, dysphagia and voice alteration $\geq$ grade 3 were considered functional defects. The grades of dysfunction in swallowing and speech were based on both clinician and patient assessments and, if available, referred to the result of verbal function tests such as the Korean version of the Boston Naming Test (K-BNT) or Motor Speech Treatment Protocol (MSTP). For patients developing subjective swallowing difficulty, the swallowing test was performed with video esophagofluoroscopy under the category of pharyngeal phase and the presence or absence of aspiration.

\section{Statistical analysis}

Statistical analysis was performed using IBM SPSS Statistics for Windows ver. 23.0 (IBM Corp., Armonk, NY). The differences in characteristics and toxicities between the two groups were compared using chi-square tests, and linearby-linear association analysis was performed to calculate the linear relationship for more than two groups. We calculated survival time from the date of diagnosis until death or the most recent follow-up date. Survival curves were estimated using the Kaplan-Meier method and were compared using log-rank tests. Cox proportional hazards models were used to assess the association of variables with survival. p-values less than 0.05 were considered statistically significant. 
Table 1. Patient and treatment characteristics

\begin{tabular}{|c|c|c|c|c|}
\hline Characteristic & Total $(n=102)$ & SRT $(n=56)$ & $\mathrm{RT}(\mathrm{n}=46)$ & p-value \\
\hline Age, median (range, yr) & $58(36-96)$ & $57(36-77)$ & $60(37-96)$ & \\
\hline \multicolumn{5}{|l|}{ Age $(y r)$} \\
\hline$\leq 58$ & $52(51.0)$ & $32(57.1)$ & $20(43.5)$ & 0.170 \\
\hline$>58$ & $50(49.0)$ & $24(42.9)$ & $26(56.5)$ & \\
\hline \multicolumn{5}{|l|}{ Sex } \\
\hline Female & $17(16.7)$ & $13(23.2)$ & $4(8.7)$ & 0.050 \\
\hline Male & $85(83.3)$ & $43(76.8)$ & $42(91.3)$ & \\
\hline \multicolumn{5}{|l|}{ Performance status } \\
\hline ECOG PS 0-1 & $92(90.2)$ & $54(96.4)$ & $38(82.6)$ & 0.040 \\
\hline ECOG PS 2 & $10(9.8)$ & $2(3.6)$ & $8(17.4)$ & \\
\hline \multicolumn{5}{|l|}{ T stage } \\
\hline 1 & $27(26.5)$ & $18(32.1)$ & 9 (19.6) & 0.029 \\
\hline 2 & $28(27.5)$ & $18(32.1)$ & $10(21.7)$ & \\
\hline 3 & $19(18.6)$ & $8(14.3)$ & $11(23.9)$ & \\
\hline $4 a$ & $27(26.5)$ & $12(21.4)$ & $15(32.6)$ & \\
\hline $4 b$ & $1(1.0)$ & 0 & $1(2.2)$ & \\
\hline \multicolumn{5}{|l|}{ N stage } \\
\hline 0 & $15(14.7)$ & $7(12.5)$ & $8(17.4)$ & 0.090 \\
\hline 1 & $19(18.6)$ & $15(26.8)$ & $4(8.7)$ & \\
\hline $2 \mathrm{a}$ & $7(6.9)$ & $5(8.9)$ & $2(4.3)$ & \\
\hline $2 b$ & $45(44.1)$ & $25(44.6)$ & $20(43.5)$ & \\
\hline $2 c$ & $16(15.7)$ & $4(7.1)$ & $12(26.1)$ & \\
\hline \multicolumn{5}{|l|}{ Stage } \\
\hline I & $3(2.9)$ & $1(1.8)$ & $2(4.3)$ & 0.393 \\
\hline II & $7(6.9)$ & $5(8.9)$ & $2(4.3)$ & \\
\hline III & $14(13.7)$ & $10(17.9)$ & $4(8.7)$ & \\
\hline IVA & $77(75.5)$ & $40(71.4)$ & $37(80.4)$ & \\
\hline IVB & $1(1.0)$ & 0 & $1(2.2)$ & \\
\hline \multicolumn{5}{|l|}{ p16 } \\
\hline$(-)$ & $17(29.3)$ & $11(28.9)$ & $6(30.0)$ & 0.933 \\
\hline$(+)$ & $41(70.7)$ & $27(71.1)$ & $14(70.0)$ & \\
\hline RT dose, mean (range, Gy) & $64.9(52.0-76.0)$ & $61.5(52.0-68.4)$ & $69.0(63.0-76.0)$ & $<0.001$ \\
\hline \multicolumn{5}{|l|}{ RT modality } \\
\hline 3D CRT & $29(28.4)$ & $16(28.6)$ & $13(28.3)$ & 0.972 \\
\hline IMRT & $73(71.6)$ & $40(71.4)$ & $33(71.7)$ & \\
\hline \multicolumn{5}{|l|}{ Chemotherapy } \\
\hline No chemotherapy & $39(38.2)$ & $35(62.5)$ & $4(8.7)$ & $<0.001$ \\
\hline Concurrent & $49(48)$ & $19(33.9)$ & $30(65.2)$ & \\
\hline Induction+concurrent & $14(13.7)$ & $2(3.6)$ & $12(26.1)$ & \\
\hline \multicolumn{5}{|l|}{ Chemotherapy regimen } \\
\hline Erbitux based & $6(9.4)$ & 0 & $6(14.3)$ & 0.163 \\
\hline Platinum based & $57(89.1)$ & $22(100)$ & 35 (83.3) & \\
\hline Both & $1(1.5)$ & 0 & $1(2.4)$ & \\
\hline
\end{tabular}

Values are presented as number $(\%)$ unless otherwise indicated. SRT, surgery followed by radiotherapy; RT, definitive radiotherapy; ECOG PS, Eastern Cooperative Oncology Group performance status; RT dose, radiotherapy dose; RT modality, radiotherapy modality; 3D CRT, 3-dimensional conformal radiotherapy; IMRT, intensity-modulated radiotherapy. 


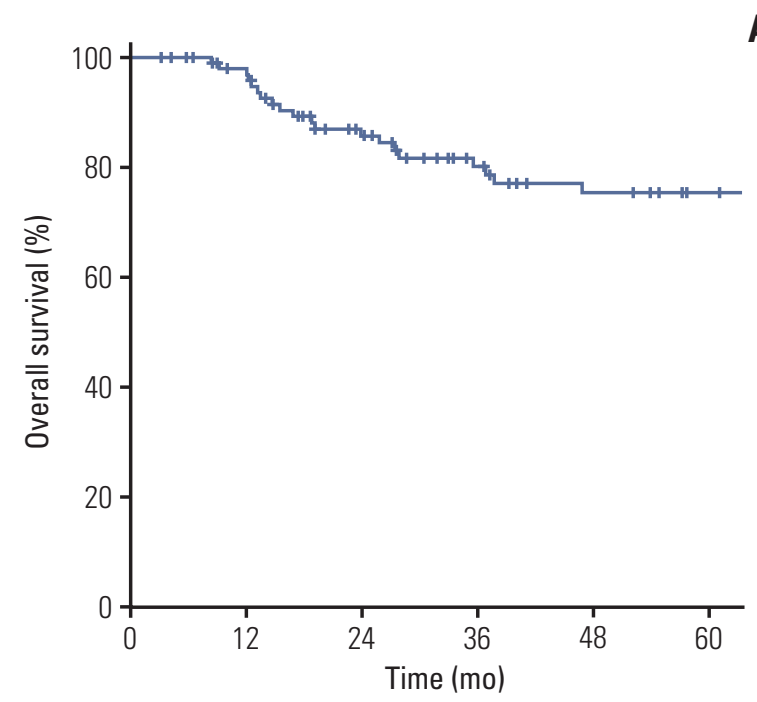

No. at risk $\begin{array}{lllll}102 & 93 & 69 & 53 & 45\end{array}$
A

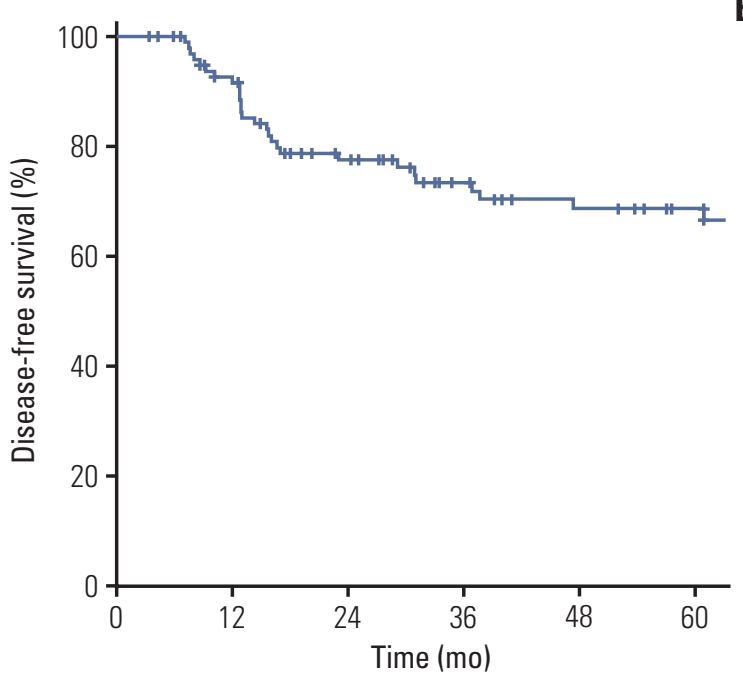

No. at risk

Fig. 1. Five-year overall survival (A) and disease-free survival (B) for the entire study cohort.

\section{Results}

\section{Patient and treatment characteristics}

Table 1 shows the patient and treatment characteristics. The median age of the patients was 58 years (range, 36 to 96 years). A total of 92 patients $(90.2 \%)$ had stage III-IV disease, with $47(46.1 \%)$ having T3-4 and $87(85.3 \%)$ having nodal positive (N1-3) disease. p16 expression status was available in 58 patients $(56.9 \%)$. Among these, $41(70.7 \%)$ had p16-positive and $17(29.3 \%)$ had p16-negative tumors.

The characteristics that differed by treatment group included sex, initial Eastern Cooperative Oncology Group performance status (ECOG PS), T stage, RT dose, and chemotherapy. More patients in the RT group were male $(91.3 \%$ vs. $76.8 \%, \mathrm{p}=0.050)$, ECOG PS $\geq 2$ (17.4\% vs. 3.6\%, $\mathrm{p}=0.040)$, had T3-4 disease (58.7\% vs. $35.7 \%, \mathrm{p}=0.021)$, and received concurrent chemotherapy $(91.3 \%$ vs. $37.5 \%$, p < 0.001$)$ compared to the SRT group. The mean RT dose was higher in the RT group (69.0 Gy vs. 61.5 Gy, p < 0.001). The other characteristics were well balanced between the two groups.

A total of 56 patients received surgery on the primary tumor. Among these, $20(35.7 \%)$ were treated via TORS and five $(9 \%)$ underwent total glossectomy. Bilateral $(n=24)$ or ipsilateral $(n=36)$ neck dissection was performed in 60 patients, including those with elective neck dissection after definitive RT $(n=5)$.

\section{Survival outcome and prognostic factors}

At a median follow-up of 36.9 months (range, 3.3 to 181.5 months), the overall Kaplan-Meier estimations of the 5-year overall survival (OS) and disease-free survival (DFS) rates for all patients were $75.5 \%$ and $68.7 \%$, respectively (Fig. 1). In univariate analysis, performance status (OS, $\mathrm{p}=0.010$; DFS, $\mathrm{p}=0.014)$, $\mathrm{T}$ stage (OS, $\mathrm{p}=0.022 ; \mathrm{DFS}, \mathrm{p}=0.005)$, RT modality (OS, $\mathrm{p}=0.005$; DFS, $\mathrm{p}=0.006$ ), and $\mathrm{p} 16$ expression (OS, $\mathrm{p}=0.003$; DFS, $\mathrm{p}<0.001$ ) were identified as prognostic factors related to both OS and DFS (Table 2). In multivariate analysis, p16 expression was an independent factor for both OS and DFS (p16-negative vs. p16-positive; OS: hazard ratio [HR], 0.145; 95\% confidence interval [CI], 0.025 to 0.853 ; $\mathrm{p}=0.033$; DFS: HR, $0.164 ; 95 \% \mathrm{CI}, 0.045$ to $0.598 ; \mathrm{p}=0.006$ ) (Table 3).

\section{Outcome comparisons according to treatment group}

There were no significant differences in the 5-year OS (68.7\% vs. $80.5 \%, \mathrm{p}=0.601)$ and DFS (63.1\% vs. $73.1 \%$, $\mathrm{p}=0.653$ ) between treatment groups (Fig. 2).

In the subgroup analysis of T3-4 patients, the 5-year OS (58.1\% vs. $64.9 \%, \mathrm{p}=0.906)$ and DFS (48\% vs. $63.5 \%, \mathrm{p}=0.958)$ showed no significant difference according to the treatment group (Fig. 3).

Data were analyzed separately for 38 patients with p16positive disease. There was no significant difference in the 5-year OS (100\% vs. $93.8 \%, \mathrm{p}=0.342)$ and DFS (90\% vs. $88 \%$, 
Table 2. Univariate analysis for OS and DFS

\begin{tabular}{|c|c|c|c|c|c|c|c|}
\hline \multirow[b]{2}{*}{ Variable } & \multirow{2}{*}{$\begin{array}{c}\text { No. of } \\
\text { patients }(\%)\end{array}$} & \multicolumn{3}{|c|}{ OS } & \multicolumn{3}{|c|}{ DFS } \\
\hline & & $\begin{array}{c}\text { 5-Year } \\
(\%)\end{array}$ & $\begin{array}{c}\text { HR } \\
(95 \% \text { CI })\end{array}$ & p-value & $\begin{array}{c}\text { 5-Year } \\
(\%)\end{array}$ & $\begin{array}{c}\text { HR } \\
(95 \% \mathrm{CI})\end{array}$ & p-value \\
\hline \multicolumn{8}{|l|}{ Age (yr) } \\
\hline$\leq 58$ & $52(51.0)$ & 83.5 & $1.573(0.707-3.501)$ & 0.267 & 72.5 & $1.275(0.634-2.564)$ & 0.496 \\
\hline$>58$ & $50(49.0)$ & 67.2 & & & 65.0 & & \\
\hline \multicolumn{8}{|l|}{ Sex } \\
\hline Female & $17(16.7)$ & 92.3 & $2.935(0.689-12.500)$ & 0.145 & 84.6 & $3.718(0.887-15.589)$ & 0.073 \\
\hline Male & $85(83.3)$ & 72.1 & & & 65.6 & & \\
\hline \multicolumn{8}{|l|}{ Performance status } \\
\hline ECOG PS 0-1 & $92(90.2)$ & 79.6 & 3.698 (1.369-9.987) & 0.010 & 72.8 & $3.090(1.260-7.579)$ & 0.014 \\
\hline ECOG PS 2 & $10(9.8)$ & 36.0 & & & 30.5 & & \\
\hline \multicolumn{8}{|l|}{ T stage } \\
\hline T1-T2 & $55(53.9)$ & 89.2 & $2.671(1.150-6.202)$ & 0.022 & 81.7 & $2.963(1.398-6.281)$ & 0.005 \\
\hline T3-T4a/b & $47(46.1)$ & 61.3 & & & 55.0 & & \\
\hline \multicolumn{8}{|l|}{ N stage } \\
\hline N0 & $15(14.7)$ & 82.5 & $1.036(0.354-3.031)$ & 0.948 & 74.3 & $1.346(0.472-3.840)$ & 0.579 \\
\hline N1-3 & $87(85.3)$ & 74.1 & & & 67.6 & & \\
\hline \multicolumn{8}{|l|}{ Stage } \\
\hline I-II & $10(9.8)$ & 100.0 & $2.653(0.359-19.621)$ & 0.339 & 80.0 & $3.363(0.459-24.655)$ & 0.233 \\
\hline III-IV & $92(90.2)$ & 73.2 & & & 67.3 & & \\
\hline \multicolumn{8}{|l|}{ p16 } \\
\hline$(-)$ & $17(29.3)$ & 60.7 & $0.088(0.017-0.450)$ & 0.003 & 46.5 & $0.119(0.036-0.391)$ & $<0.001$ \\
\hline$(+)$ & $41(70.7)$ & 95.2 & & & 88.3 & & \\
\hline \multicolumn{8}{|l|}{ Treatment } \\
\hline SRT & $56(54.9)$ & 80.5 & $1.233(0.562-2.704)$ & 0.602 & 73.1 & $1.173(0.585-2.351)$ & 0.654 \\
\hline RT & $46(45.1)$ & 68.7 & & & 63.1 & & \\
\hline RT dose & & & $0.995(0.943-1.050)$ & 0.859 & & $0.994(0.948-1.042)$ & 0.808 \\
\hline \multicolumn{8}{|l|}{ RT modality } \\
\hline 3D CRT & $29(28.4)$ & 62.1 & $0.304(0.133-0.697)$ & 0.005 & 51.7 & $0.369(0.183-0.748)$ & 0.006 \\
\hline IMRT & $73(71.6)$ & 81.4 & & & 77.4 & & \\
\hline \multicolumn{8}{|l|}{ Chemotherapy } \\
\hline Not concurrent & $39(38.2)$ & 74.3 & $0.750(0.341-1.653)$ & 0.476 & 69.6 & $0.882(0.439-1.772)$ & 0.725 \\
\hline Concurrent & $63(61.8)$ & 76.3 & & & 68.2 & & \\
\hline
\end{tabular}

OS, overall survival; DFS, disease-free survival; HR, hazard ratio; CI, confidence interval; ECOG PS, Eastern Cooperative Oncology Group performance status; SRT, surgery followed by radiotherapy; RT, definitive radiotherapy; RT dose, radiotherapy dose; RT modality, radiotherapy modality; 3D CRT, 3-dimensional conformal radiotherapy; IMRT, intensity-modulated radiotherapy.

$\mathrm{p}=0.865$ ) between the RT and SRT groups (Fig. 4).

\section{Patterns of failure}

During the follow-up period, treatment failure occurred in 10 and 13 patients in the RT and SRT groups, respectively. Table 4 shows the patterns of failure and outcomes of salvage treatment among the patients who experienced local and/or regional failures. Among eight failures in the RT group, only one patient received re-irradiation with concurrent chemo- therapy; two were salvaged with neck dissection and two with chemotherapy as the sole treatment. All of these patients died of disease (survival after recurrence, 3 to 15 months) except for one patient who was successfully salvaged with neck dissection (survival after recurrence, 4 months). Among six failures in the SRT group, three patients were salvaged with chemotherapy or re-irradiation and two underwent surgical resection of recurrent primary tumors. Four of those who received salvage treatment died of disease progression (survival after recurrence, 3 to 16 months) and 
Table 3. Multivariate analysis for OS and DFS

\begin{tabular}{|c|c|c|c|c|c|c|c|}
\hline \multirow[b]{2}{*}{ Variable } & \multirow{2}{*}{$\begin{array}{c}\text { No. of } \\
\text { patients }(\%)\end{array}$} & \multicolumn{3}{|c|}{ OS } & \multicolumn{3}{|c|}{ DFS } \\
\hline & & $\begin{array}{c}\text { 5-Year } \\
(\%)\end{array}$ & $\begin{array}{c}\text { HR } \\
(95 \% \mathrm{CI})\end{array}$ & p-value & $\begin{array}{c}\text { 5-Year } \\
(\%)\end{array}$ & $\begin{array}{c}\text { HR } \\
(95 \% \mathrm{CI})\end{array}$ & p-value \\
\hline \multicolumn{8}{|c|}{ Performance status } \\
\hline ECOG PS 0-1 & $53(91.4)$ & 91.2 & $6.465(0.346-120.758)$ & 0.211 & 82.3 & $2.507(0.401-15.666)$ & 0.326 \\
\hline ECOG PS 2 & $5(8.6)$ & 37.5 & & & 26.7 & & \\
\hline \multicolumn{8}{|l|}{ T stage } \\
\hline T1-T2 & $34(58.6)$ & 92.3 & $0.785(0.129-4.780)$ & 0.793 & 82.3 & $1.146(0.313-4.202)$ & 0.837 \\
\hline $\mathrm{T} 3-\mathrm{T} 4 \mathrm{a} / \mathrm{b}$ & $24(41.4)$ & 79.5 & & & 69.5 & & \\
\hline \multicolumn{8}{|l|}{ p16 } \\
\hline$(-)$ & $17(29.3)$ & 60.7 & $0.145(0.025-0.853)$ & 0.033 & 46.5 & $0.164(0.045-0.598)$ & 0.006 \\
\hline$(+)$ & $41(70.7)$ & 95.2 & & & 88.3 & & \\
\hline \multicolumn{8}{|l|}{ RT modality } \\
\hline 3D CRT & $11(19.0)$ & 81.8 & $0.237(0.022-2.505)$ & 0.231 & 72.7 & $0.489(0.116-2.057)$ & 0.326 \\
\hline IMRT & $47(81.0)$ & 85.6 & & & 78.2 & & \\
\hline
\end{tabular}

OS, overall survival; DFS, disease-free survival; HR, hazard ratio; CI, confidence interval; ECOG PS, Eastern Cooperative Oncology Group performance status; RT modality, radiotherapy modality; 3D CRT, 3-dimensional conformal radiotherapy; IMRT, intensity-modulated radiotherapy.

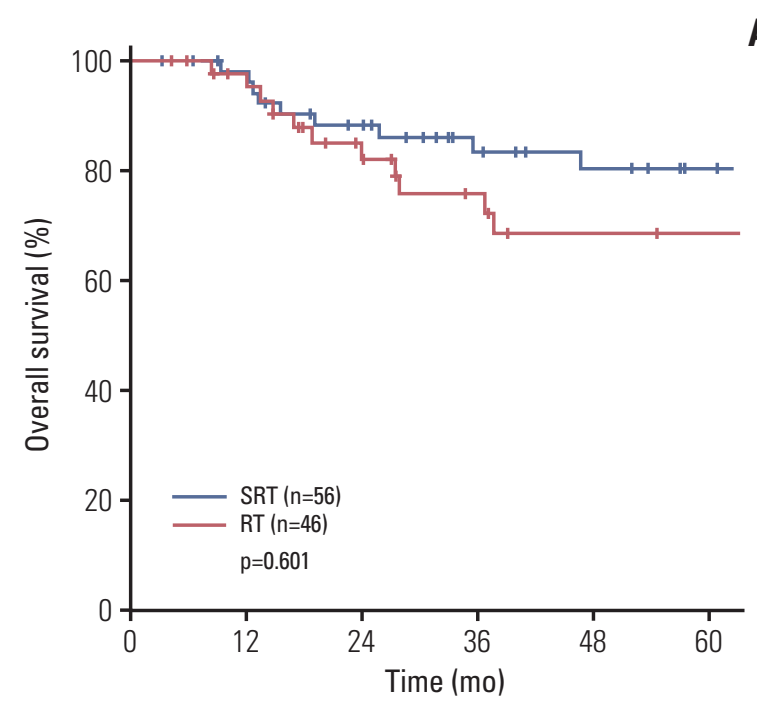

A

No. at risk

\begin{tabular}{|c|c|c|c|c|c|}
\hline SRT & 56 & 52 & 41 & 31 & 27 \\
\hline RT & 46 & 41 & 28 & 22 & 18 \\
\hline
\end{tabular}

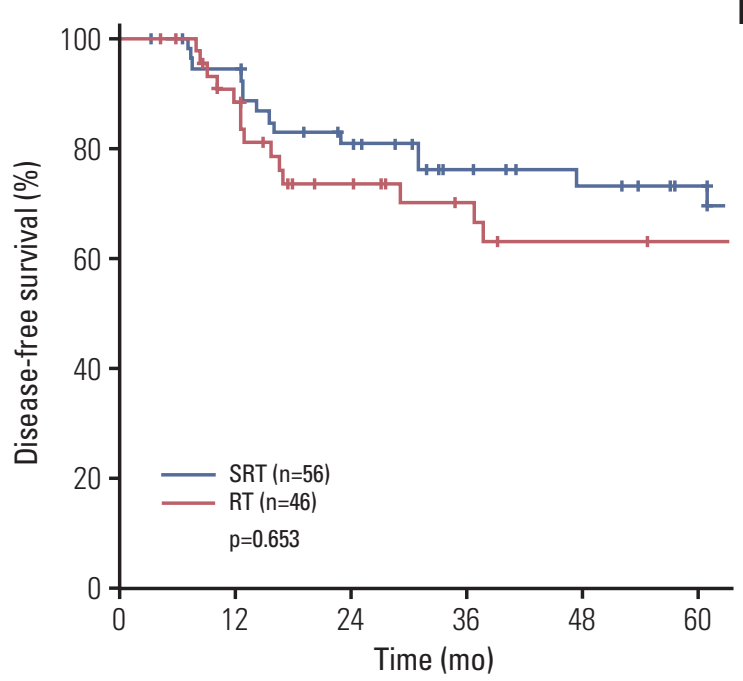

Fig. 2. Comparison of 5-year overall survival (A) and disease-free survival (B) between the definitive definitive radiotherapy (RT) and surgery followed by radiotherapy (SRT) groups.

another died of bleeding after salvage surgery (survival after recurrence, 3 months).

\section{Toxicities and functional outcomes}

The complications requiring surgical intervention are described in Table 5. Wound dehiscence, bleeding, infection, 


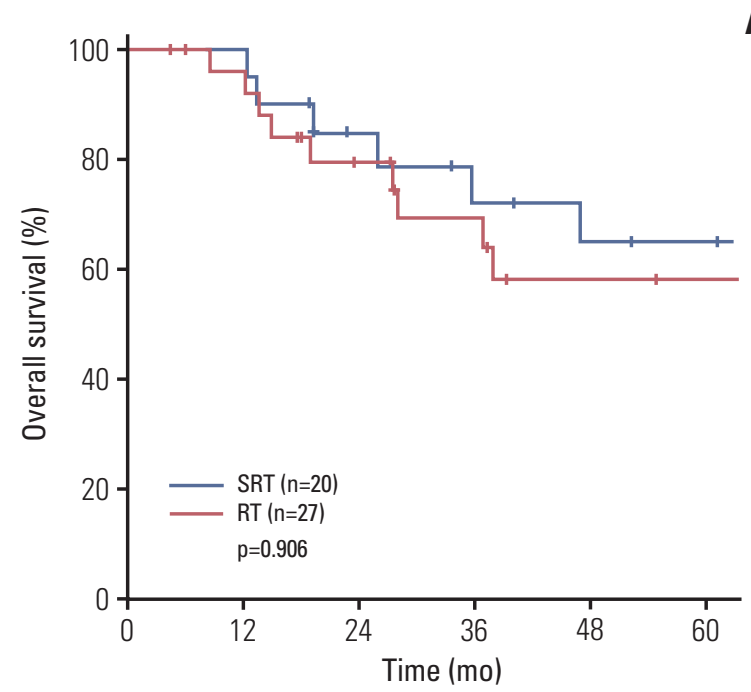

A

B

No. at risk

$\begin{array}{rrrrrrr}\text { SRT } & 20 & 20 & 14 & 11 & 9 & 8 \\ \text { RT } & 27 & 24 & 17 & 13 & 9 & 8\end{array}$

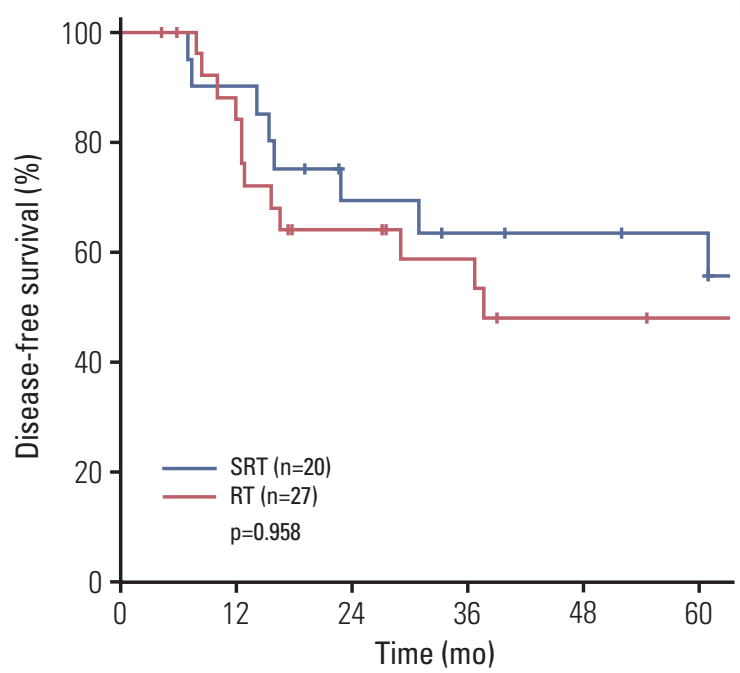

No. at risk

$\begin{array}{rrrrrrr}\text { SRT } & 20 & 18 & 12 & 10 & 9 & 8 \\ \text { RT } & 27 & 21 & 14 & 11 & 8 & 7\end{array}$

Fig. 3. Comparison of 5-year overall survival (A) and disease-free survival (B) between treatment groups in T3-4 patients. SRT, surgery followed by radiotherapy; RT, definitive radiotherapy.

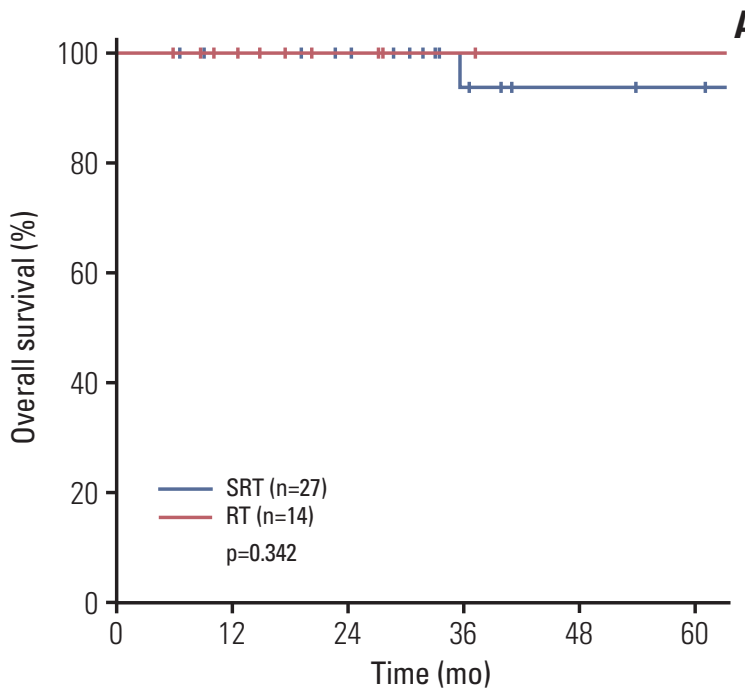

A

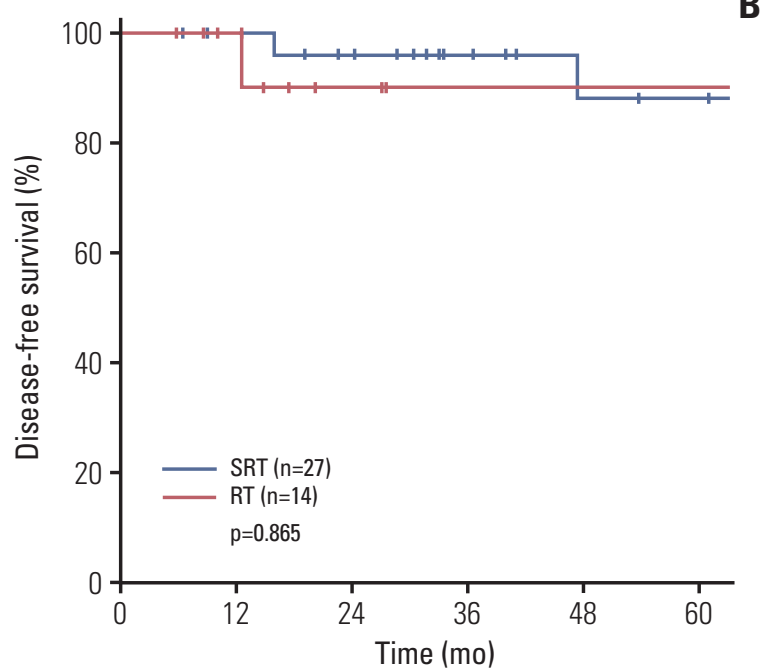

No. at risk

$\begin{array}{rrrrrrr}\text { SRT } & 27 & 25 & 22 & 15 & 12 & 11 \\ \text { RT } & 14 & 11 & 7 & 5 & 4 & 4\end{array}$

No. at risk

$\begin{array}{rrrrrrr}\text { SRT } & 27 & 25 & 21 & 15 & 11 & 10 \\ \text { RT } & 14 & 11 & 6 & 4 & 4 & 4\end{array}$

Fig. 4. Comparison of 5-year overall survival (A) and disease-free survival (B) between treatment groups in p16-positive patients. SRT, surgery followed by radiotherapy; RT, definitive radiotherapy. 


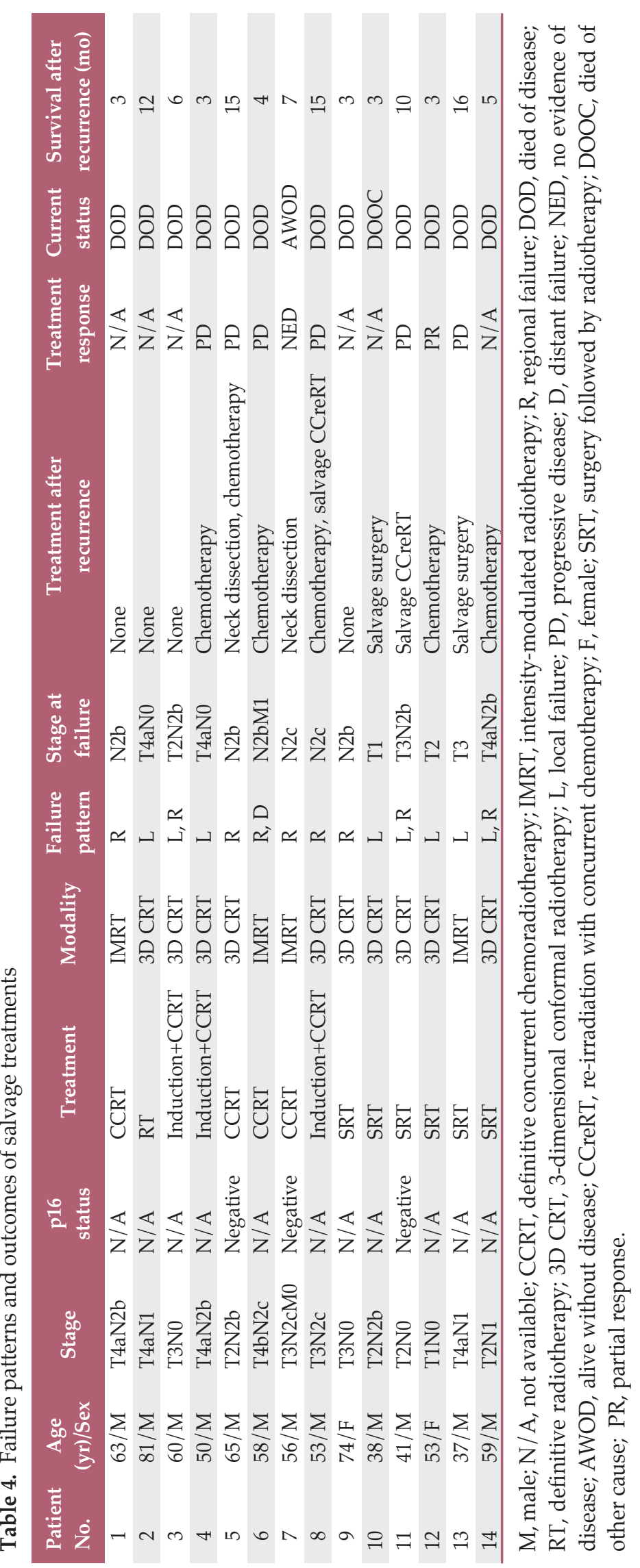


Table 5. Complications requiring surgical intervention

\begin{tabular}{|c|c|c|c|c|c|c|}
\hline \multirow{2}{*}{ Variable } & \multirow{2}{*}{$\begin{array}{c}\text { Total } \\
(\mathrm{n}=102)\end{array}$} & \multicolumn{3}{|c|}{ SRT $(n=56)$} & \multirow{2}{*}{$\begin{array}{c}\mathrm{RT} \\
(\mathrm{n}=46)\end{array}$} & \multirow{2}{*}{ p-value ${ }^{a}$} \\
\hline & & Total $(n=56)$ & Open $(n=36)$ & TORS $(n=20)$ & & \\
\hline Wound infection & $1(1.0)$ & $1(1.8)$ & $1(2.8)$ & 0 & 0 & 0.001 \\
\hline Wound dehiscence & $5(4.9)$ & $4(7.1)$ & $2(5.6)$ & $2(10.0)$ & $1(2.2)$ & \\
\hline Wound bleeding & $4(3.9)$ & $4(7.1)$ & $3(8.3)$ & $1(5.0)$ & 0 & \\
\hline Wound fistula & $2(2.0)$ & $2(3.6)$ & $2(5.6)$ & 0 & 0 & \\
\hline Pharyngeal stenosis & $1(1.0)$ & $1(1.8)$ & $1(2.8)$ & 0 & 0 & \\
\hline Flap necrosis & $5(4.9)$ & $5(8.9)$ & $5(13.9)$ & 0 & 0 & \\
\hline Scar contracture & $3(2.9)$ & $3(5.4)$ & $3(8.3)$ & 0 & 0 & \\
\hline
\end{tabular}

Values are presented as number (\%). SRT, surgery followed by radiotherapy; RT, definitive radiotherapy; Open, open surgery group; TORS, trans-oral robotic surgery group. ${ }^{\text {a) }}$-value was calculated between SRT group and RT group.

Table 6. Toxicities and pharyngeal function after treatment

\begin{tabular}{|c|c|c|c|c|c|c|c|}
\hline \multirow{2}{*}{ Toxicity } & \multirow{2}{*}{ Grade } & \multirow{2}{*}{$\begin{array}{c}\text { Total } \\
(n=102)\end{array}$} & \multicolumn{3}{|c|}{ SRT $(n=56)$} & \multirow{2}{*}{$\begin{array}{c}\text { RT } \\
(n=46)\end{array}$} & \multirow{2}{*}{ p-value } \\
\hline & & & Total $(n=56)$ & Open $(n=36)$ & TORS $(n=20)$ & & \\
\hline \multicolumn{8}{|l|}{ Acute } \\
\hline Skin & G3 & $8(7.8)$ & $1(1.8)$ & 0 & $1(5.0)$ & $7(15.2)$ & 0.271 \\
\hline Mucositis & G3 & $21(20.6)$ & $8(14.3)$ & $3(8.3)$ & $5(25.0)$ & $13(28.3)$ & \\
\hline Fatigue & G3 & $1(1.0)$ & 0 & 0 & 0 & $1(2.2)$ & \\
\hline \multirow[t]{2}{*}{ Voice alteration } & G3 & $1(1.0)$ & $1(1.8)$ & $1(2.8)$ & 0 & 0 & \\
\hline & G4 & $5(4.9)$ & $5(8.9)$ & $5(13.9)$ & 0 & 0 & \\
\hline Dysphagia & G3 & $4(3.9)$ & $4(7.1)$ & $2(5.6)$ & $2(10.0)$ & 0 & \\
\hline \multicolumn{8}{|l|}{ Late } \\
\hline Xerostomia & G3 & $1(1.0)$ & $1(1.8)$ & $1(2.8)$ & 0 & 0 & 0.006 \\
\hline Osteoradionecrosis & G3 & $3(2.9)$ & $3(5.4)$ & $2(5.6)$ & $1(5.0)$ & 0 & \\
\hline Dysphagia & G3 & $3(2.9)$ & $3(5.4)$ & $3(8.3)$ & 0 & 0 & \\
\hline \multirow[t]{2}{*}{ Voice alteration } & G3 & $5(4.9)$ & $4(7.1)$ & $3(8.3)$ & $1(5.0)$ & $1(2.2)$ & \\
\hline & G4 & $5(4.9)$ & $5(8.9)$ & $5(13.9)$ & 0 & 0 & \\
\hline \multicolumn{8}{|l|}{ Functional defect } \\
\hline Dysphagia & $\geq \mathrm{G} 3$ & $3(2.9)$ & $3(5.4)$ & $3(8.3)$ & 0 & 0 & 0.250 \\
\hline Voice alteration & $\geq \mathrm{G} 3$ & $10(9.8)$ & 9 (16.1) & $8(22.2)$ & $1(5.0)$ & $1(2.2)$ & 0.021 \\
\hline
\end{tabular}

Values are presented as number (\%). SRT, surgery followed by radiotherapy; RT, definitive radiotherapy; Open, open surgery group; TORS, trans-oral robotic surgery group; G, grade. a)p-value was calculated between SRT group and RT group.

and flap necrosis were among the immediate complications, whereas wound fistula, pharyngeal stenosis, and scar contracture were considered delayed complications. The wound complications were more frequent in the SRT group than in the RT group ( $25 \%$ vs. $2.2 \%$, $\mathrm{p}=0.001$ ).

Table 6 shows the acute and late toxicities in accordance with CTCAE ver. 4.0. There was no significant difference in the rates of overall $\geq$ grade 3 acute toxicities between treatment groups $(28.6 \%$ vs. $37 \%, p=0.271)$, while $\geq$ grade 3 late toxicities were more frequently observed in the SRT group (20\% vs. 2.2\%, p=0.006). Functional defects (defined as late grade $\geq 3$ dysphagia or voice alteration) were more frequently reported in the SRT group than in the RT group (16.1\% vs. $2.2 \%, \mathrm{p}=0.021)$.

In surgery group, patients were sub-classified to open surgery group and TORS group. The complications requiring surgical intervention more frequently occurred in open surgery group than TORS group. Similarly, open surgery group reported more severe treatment-related late toxicities and functional defect compared to TORS group. When comparing the function of TORS group with that of RT group, only one patient $(5 \%)$ reported severe dysfunction and no signif- 
icant difference was noted compared to the RT group (TORS group vs. RT group, $5 \%$ vs. $2.2 \%$, $\mathrm{p}=0.517$ ).

\section{Discussion}

BOT cancer is a relatively rare disease, with a reported agestandardized incidence rate of 0.5/100,000 person-year in developed countries [8], though it is the second most common primary site in oropharyngeal cancer (OPC). The prognosis of BOT cancer is worse than that of tonsillar cancer, the most common OPC [9]. However, until now, most evidence has been provided by studies of OPC, which include more cases of primary tonsillar cancer than primary BOT cancer. Several studies have reported treatment results only for BOT cancer but did not directly compare treatment outcomes between surgery and RT.

Historically, surgical treatment, which had been considered the preferred treatment, had the advantage of being able to remove the tumor curatively; however, it reportedly causes serious dysfunction and frequent loco-regional recurrence. Surgery alone is considered an appropriate treatment for early-stage disease, but treatment outcomes for locally advanced disease were not satisfactory [2,3]. To achieve both tumor control and functional preservation, definitive RT was considered as primary treatment. Several studies reported favorable outcomes after definitive RT when compared to the historical results of primary surgery [10-12]. However, although offering better functional preservation, external beam RT alone showed poor oncologic outcomes in locally advanced stage disease $[10,13]$. Consequently, to improve tumor control, several methods have been investigated, including the concurrent use of chemotherapy for radiosensitization [14], altered daily fractionation with hyperfractionation [15], interstitial brachytherapy, and external beam RT boost $[1,16]$.

Recently, minimally-invasive transoral endoscopic surgery (TES) has made it possible to treat OPC with better functional preservation than that of traditional open approaches. Among TES approaches, transoral laser microsurgery showed excellent oncological and functional outcome in head and neck cancer [17]; a more recent technique, TORS has demonstrated its feasibility for the removal of the tumors in oropharyngeal region [18]. In addition, the dose conformality of radiation therapy has improved and the introduction of IMRT has made it possible to deliver the higher doses needed to control gross tumors while preserving unaffected organs near the target.

Another remarkable finding in cancer biology was the report that human papillomavirus (HPV)-positive OPC has a quite different etiology and prognosis from those of HPVnegative counterparts [19]. Although not yet fully explored, HPV-associated tumors are suspected to be caused by E6 and E7 viral oncoproteins that bind to and consequently inactivate the $\mathrm{p} 53$ tumor suppressor gene and retinoblastoma protein $(\mathrm{pRB})$, resulting in the malignant transformation of HPV-infected cells. As a result, the biology of HPV-positive tumors is distinct from that of HPV-negative tumors, featuring p16 up-regulation due to reduced negative feedback from the pRB pathway [20-22]. The better prognosis of HPVpositive tumors was noted after treatment including RT as well as surgery $[23,24]$. A possible explanation for the improved survival in HPV-positive tumors after RT might be the functional TP53 presence in HPV-positive tumor, which makes the tumor more susceptible to radiation-induced apoptosis, in contrast to HPV-negative, tobacco-associated tumors [25]. The functional preservation of the pharynx and tongue is especially important for patients with HPV-positive OPCs who are expected to survive longer. However, studies on the survival and functional outcomes of BOT cancer lack data on HPV infection status. In the current study, multivariate analysis revealed that $\mathrm{p} 16$ expression status, in other words, HPV infection status, was the only independent factor for survival.

This study compared the treatment and functional outcomes of patients treated with definitive RT or surgery followed by adjuvant RT with modern techniques in the same institution. In addition, the HPV infection status was reported in more than half of the patients. A comparison between the two treatment groups showed that the patient and treatment characteristics did not differ significantly except for the proportion of locally advanced disease, the proportion of patients with poor performance status, the proportion of men, and the use of chemotherapy, which were higher in the RT group. The higher proportion of advanced disease and poor performance status in the RT group could be explained by the fact that curative resection is not feasible for these patients; in these cases, definitive RT might be preferred. The addition of concurrent chemotherapy to RT was proven to be superior to RT alone in the early 2000s [26,27]; we have used concurrent chemotherapy routinely during definitive RT with some exception (e.g., old age, medical condition, patient denial). However, the benefit of chemotherapy during adjuvant RT is not clear except for cases with adverse pathologic features $[28,29]$. Therefore, patients undergoing surgery in our institution received chemotherapy only if adverse features were reported. When comparing the treatment outcomes of the two groups, there was no statistically significant difference in OS or DFS. In addition, subgroup analysis of patients with T3-4 and HPV-positive disease did not reveal any significant differences between the two treatment groups. These results suggest the possibility that RT 
could be considered as the preferred treatment for patients with locally advanced stage disease expected to have severe morbidity after surgery and for patients with HPV-positive disease expected to have a favorable prognosis regardless of treatment modality. Regarding toxicities and functional outcomes, late toxicities above grade 3 occurred more frequently in the SRT group and voice alteration above grade 3 was also frequently reported in the SRT group, which implies that better functional preservation is expected by definitive RT compared to surgery, even in more advanced disease. However, when SRT group was sub-classified into open surgery group and TORS group, the wound complications, late toxicities were less frequently reported in TORS group than open surgery group, and the functional outcomes of TORS group were comparable to the RT group. Nevertheless, caution should be exercised before deriving any conclusions based on these findings, because only 20 patients underwent TORS in our cohort; among them, 16 had T1-2 disease.

Several limitations should be taken into account, stemming from the retrospective nature of this study. The major limitation was the heterogeneous radiation doses and chemotherapy regimens. The radiation doses differed considerably within both treatment groups (SRT group, 52-68.4 Gy; RT group, 63-76 Gy). Additionally, the chemotherapy schedules and regimen themselves were heterogeneous. In addition, our study cohort was relatively small, as the incidence of primary BOT cancer is low.

Despite these limitations, in the absence of a randomized controlled trial with large population of BOT cancer, this is one of the largest single-institution studies to compare patients treated with primary surgery and definitive RT treated contemporarily. The results of current study suggest that definitive RT showed no significant differences in survival, with better function when compared to surgery followed by postoperative RT, despite the fact that the proportion of patients with locally advanced stage disease was higher in the RT group. Definitive RT might be considered as a preferred primary treatment option, especially for HPVpositive patients who are expected to have a long-life expectancy or for those with locally advanced stage disease in which surgical resection may result in severe defects.

\section{Conflicts of Interest}

Conflict of interest relevant to this article was not reported.

\section{References}

1. Karakoyun-Celik O, Norris CM Jr, Tishler R, Mahadevan A, Clark JR, Goldberg S, et al. Definitive radiotherapy with interstitial implant boost for squamous cell carcinoma of the tongue base. Head Neck. 2005;27:353-61.

2. Sessions DG, Lenox J, Spector GJ, Chao C, Chaudry OA. Analysis of treatment results for base of tongue cancer. Laryngoscope. 2003;113:1252-61.

3. Machtay M, Perch S, Markiewicz D, Thaler E, Chalian A, Goldberg A, et al. Combined surgery and postoperative radiotherapy for carcinoma of the base of radiotherapy for carcinoma of the base of tongue: analysis of treatment outcome and prognostic value of margin status. Head Neck. 1997;19:494-9.

4. Fein DA, Lee WR, Amos WR, Hinerman RW, Parsons JT, Mendenhall WM, et al. Oropharyngeal carcinoma treated with radiotherapy: a 30-year experience. Int J Radiat Oncol Biol Phys. 1996;34:289-96.

5. Lorincz BB, Jowett N, Knecht R. Decision management in transoral robotic surgery: Indications, individual patient selection, and role in the multidisciplinary treatment for head and neck cancer from a European perspective. Head Neck. 2016;38 Suppl 1:E2190-6.

6. Kerr P, Myers CL, Butler J, Alessa M, Lambert P, Cooke AL. Prospective functional outcomes in sequential population based cohorts of stage III/ IV oropharyngeal carcinoma patients treated with 3D conformal vs. intensity modulated radiotherapy. J Otolaryngol Head Neck Surg. 2015;44:17.

7. Mydlarz WK, Chan JY, Richmon JD. The role of surgery for HPV-associated head and neck cancer. Oral Oncol. 2015;51: 305-13.

8. Attner P, Du J, Nasman A, Hammarstedt L, Ramqvist T, Lindholm J, et al. The role of human papillomavirus in the increased incidence of base of tongue cancer. Int J Cancer. 2010;126:2879-84.

9. Kanakamedala M, Giri S, Hamilton RD, Vijayakumar S. Outcome analysis of squamous cell carcinomas of oropharynx based on primary site that were treated with definitive intensity modulated radiation therapy concurrently with chemotherapy. Int J Radiat Oncol Biol Phys. 2016;96:Abstr 2958.

10. Mendenhall WM, Stringer SP, Amdur RJ, Hinerman RW, Moore-Higgs GJ, Cassisi NJ. Is radiation therapy a preferred alternative to surgery for squamous cell carcinoma of the base of tongue? J Clin Oncol. 2000;18:35-42.

11. Parsons JT, Mendenhall WM, Stringer SP, Amdur RJ, Hinerman RW, Villaret DB, et al. Squamous cell carcinoma of the oropharynx: surgery, radiation therapy, or both. Cancer. 2002;94:2967-80.

12. Mendenhall WM, Morris CG, Amdur RJ, Hinerman RW, Werning JW, Villaret DB. Definitive radiotherapy for squa- 
mous cell carcinoma of the base of tongue. Am J Clin Oncol. 2006;29:32-9.

13. Brunin F, Mosseri V, Jaulerry C, Point D, Cosset JM, Rodriguez J. Cancer of the base of the tongue: past and future. Head Neck. 1999;21:751-9.

14. Denis F, Garaud P, Bardet E, Alfonsi M, Sire C, Germain T, et al. Final results of the 94-01 French Head and Neck Oncology and Radiotherapy Group randomized trial comparing radiotherapy alone with concomitant radiochemotherapy in advanced-stage oropharynx carcinoma. J Clin Oncol. 2004;22: 69-76.

15. Hinerman RW, Parsons JT, Mendenhall WM, Stringer SP, Cassisi NJ, Million RR. External beam irradiation alone or combined with neck dissection for base of tongue carcinoma: an alternative to primary surgery. Laryngoscope. 1994;104: 1466-70.

16. Lawson JD, Otto K, Chen A, Shin DM, Davis L, Johnstone PA. Concurrent platinum-based chemotherapy and simultaneous modulated accelerated radiation therapy for locally advanced squamous cell carcinoma of the tongue base. Head Neck. 2008;30:327-35.

17. Steiner W, Fierek O, Ambrosch P, Hommerich CP, Kron M. Transoral laser microsurgery for squamous cell carcinoma of the base of the tongue. Arch Otolaryngol Head Neck Surg. 2003;129:36-43.

18. van Loon JW, Smeele LE, Hilgers FJ, van den Brekel MW. Outcome of transoral robotic surgery for stage I-II oropharyngeal cancer. Eur Arch Otorhinolaryngol. 2015;272:175-83.

19. Shi A, Zhu G, Wu H, Yu R, Li F, Xu B. Analysis of clinical and dosimetric factors associated with severe acute radiation pneumonitis in patients with locally advanced non-small cell lung cancer treated with concurrent chemotherapy and intensitymodulated radiotherapy. Radiat Oncol. 2010;5:35.

20. Marur S, D'Souza G, Westra WH, Forastiere AA. HPV-associated head and neck cancer: a virus-related cancer epidemic.
Lancet Oncol. 2010;11:781-9.

21. Reimers N, Kasper HU, Weissenborn SJ, Stutzer H, Preuss SF, Hoffmann TK, et al. Combined analysis of HPV-DNA, p16 and EGFR expression to predict prognosis in oropharyngeal cancer. Int J Cancer. 2007;120:1731-8.

22. Pytynia KB, Dahlstrom KR, Sturgis EM. Epidemiology of HPV-associated oropharyngeal cancer. Oral Oncol. 2014;50: 380-6.

23. Licitra L, Perrone F, Bossi P, Suardi S, Mariani L, Artusi R, et al. High-risk human papillomavirus affects prognosis in patients with surgically treated oropharyngeal squamous cell carcinoma. J Clin Oncol. 2006;24:5630-6.

24. Fischer CA, Zlobec I, Green E, Probst S, Storck C, Lugli A, et al. Is the improved prognosis of p16 positive oropharyngeal squamous cell carcinoma dependent of the treatment modality? Int J Cancer. 2010;126:1256-62.

25. Peltenburg LT. Radiosensitivity of tumor cells: oncogenes and apoptosis. Q J Nucl Med. 2000;44:355-64.

26. Adelstein DJ, Li Y, Adams GL, Wagner H Jr, Kish JA, Ensley JF, et al. An intergroup phase III comparison of standard radiation therapy and two schedules of concurrent chemoradiotherapy in patients with unresectable squamous cell head and neck cancer. J Clin Oncol. 2003;21:92-8.

27. Forastiere AA, Goepfert H, Maor M, Pajak TF, Weber R, Morrison $\mathrm{W}$, et al. Concurrent chemotherapy and radiotherapy for organ preservation in advanced laryngeal cancer. N Engl J Med. 2003;349:2091-8.

28. Cooper JS, Pajak TF, Forastiere AA, Jacobs J, Campbell BH, Saxman SB, et al. Postoperative concurrent radiotherapy and chemotherapy for high-risk squamous-cell carcinoma of the head and neck. N Engl J Med. 2004;350:1937-44.

29. Bernier J, Domenge C, Ozsahin M, Matuszewska K, Lefebvre JL, Greiner RH, et al. Postoperative irradiation with or without concomitant chemotherapy for locally advanced head and neck cancer. N Engl J Med. 2004;350:1945-52. 